emissions of $\mathrm{CO}_{2}$ that would result.

Hausfather and colleagues developed a method for evaluating the forecasts of early climate models without penalizing the models for their inaccurate estimates of unknowable future climate drivers. The authors examined 17 projections of global mean surface temperature (GMST) from 14 models. Before applying their method, they found that 10 projections were consistent with observations. But when inaccuracies in the estimates of climate drivers were taken into account, the authors discovered that 14 projections agreed with the data. Of the three that did not, two predicted higher-than-observed surface warming and one predicted lower-than-observed warming.

Developing credible climate models through an understanding of climatically relevant processes, observations and well-formulated equations is a considerable scientific and computational challenge. The equations that describe climate are complex and require substantial computing power to solve. As a result, climate models have always been run on the fastest supercomputers available. It is especially impressive that the earliest models assessed by Hausfather et al. produced accurate GMST forecasts, given the extremely limited computing power available then compared with that used today (Fig. 1).

Although the authors' findings show that climate models can accurately predict GMST, these forecasts are insufficient for understanding and preparing for the effects of ongoing climate change. For instance, regional climate change is especially subject to unpredictable climate variability, which greatly limits forecasting potential - even on decadal timescales when the climate drivers are known ${ }^{5}$. Moreover, on the basis of GMST forecasts alone, it is hard to predict, for example: to what extent sea level will rise; how ocean acidification caused by uptake of atmospheric $\mathrm{CO}_{2}$ will influence marine ecosystems; and the frequency and magnitude of future fires, droughts and floods.

Scientists will have to continue to improve climate modelling and to increase their understanding of the effects of climate change, while keeping in mind the tension between the need for increased model resolution, greater representation of climatically relevant processes, and more simulations to characterize unpredictable climate variability. The successful forecasting of GMST by early climate models is impressive, but leaves much work to be done - as scientists, policymakers and stakeholders are all well aware.

Numerical models based on scientific equations describing the atmosphere are used daily to make decisions that save lives and money. As the climate continues to change owing largely to human activities, scientists need to use, improve and communicate the value of numerical models and the equations and knowledge that underlie them. Hausfather and colleagues' work demonstrates that the physics in climate models has been providing accurate forecasts of GMST under increasing amounts of atmospheric $\mathrm{CO}_{2}$ for decades. Such predictions are useful for estimating the maximum amount of $\mathrm{CO}_{2}$ that can be released into the atmosphere over time to keep surface warming to a specified level.

Crucially, the authors' results also show that a major source of uncertainty in GMST forecasts comes from climate drivers. And, of these drivers, it is emissions of greenhouse gases from human activity that will largely determine future surface warming. The findings indicate the usefulness of climate-model predictions of GMST in response to increasing greenhouse-gas emissions, despite unknowable future climate drivers. But scientists must

also continue to develop climate models in concert with everything else available to them, to plan for a changed climate that requires much more than forecasts of surface warming.

Jennifer E. Kay is in the Department of Atmospheric and Oceanic Sciences and at the Cooperative Institute for Research in Environmental Sciences, University of Colorado at Boulder, Boulder, Colorado 80309, USA.

e-mail: jennifer.e.kay@colorado.edu

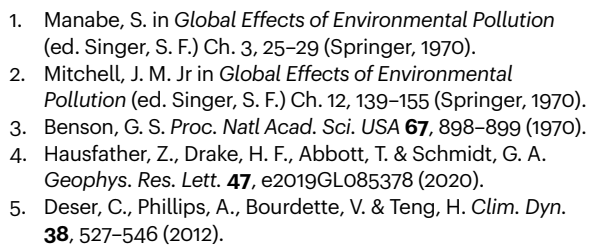

\title{
Cancer
}

\section{Brain tumours manipulate neighbouring synapses}

\author{
Nicola J. Allen
}

The growth of a brain tumour can be affected by the activity of its neighbouring neurons. The finding that such tumours send signals that boost connections between these neurons reveals a pathway that drives cancer growth. See p.166

A type of non-neuronal brain cell called a glial cell can give rise to a lethal cancer called glioblastoma ${ }^{1}$. Half of the cells in the human brain are glial cells, which normally act to support the function and communication of neurons ${ }^{2}$. Yet despite decades of research, there are no existing treatments for glioblastoma that substantially increase the survival time of people with such tumours. On page 166, Yu et al. ${ }^{3}$ report their analysis of the effects on the brain of certain glioblastoma-associated mutations. These insights might open up new strategies for anticancer research.

DNA sequencing of cancers has identified many tumour-associated mutations. However, it is a challenge to determine which of these mutations have a causal role in tumour development and growth, and which have no effect. Moreover, some mutations can be context dependent, such that the same mutation might have differing effects depending on the type of tumour and its microenvironment. It is hard to predict whether different mutations (variants) of the same gene will lead to the same outcome in different tumour types.

$\mathrm{Yu}$ and colleagues tackled these issues by studying the RTK-RAS-PI3K signalling pathway in glioblastoma. This pathway is altered in $90 \%$ of glioblastomas ${ }^{4,5}$, and mutations in it boost cell division and tumour growth. The authors focused on mutations in the gene encoding the enzyme PIK3CA - a pathway component that is often abnormal in human glioblastomas.

The authors generated mouse models of glioblastoma, and mutated genes in the RTKRAS-PI3K pathway using the gene-editing tool CRISPR-Cas9, which resulted in tumour growth. Yu et al. then engineered animals to express PIK3CA variants that are found in human glioblastomas (Fig. 1). This revealed that many of the tested variants made tumours more aggressive and rapidly lethal. This was the case both for known variants and for others that had not previously been associated with a role in glioblastoma.

Yu and colleagues studied whether alterations in the enzymatic activity of PIK3CA variants might explain how they accelerate glioblastoma progression. Surprisingly, the activity of PIK3CA was not always linked to an effect on tumour growth - some variants strongly increased enzyme activity, whereas others had a much milder effect. To investigate other mechanisms that might explain the effect 
on tumour growth, the authors performed RNA sequencing of tumour cells. Most PIK3CA variants had patterns of altered gene expression that were similar to each other in the animals' tumours, but different from the pattern in healthy tissue. However, two variants (named C420R and H1047R) had distinctive patterns compared with each other and with the other variants. Tumours containing either of these two variants showed abnormally altered expression of hundreds of genes that regulate synapses (structures that connect neurons). C420R and H1047R were also each associated with alterations in different categories of synapse-regulating gene.

Seizures are caused by a rise in neuronal activity and are often an early symptom of glioblastoma ${ }^{6}$. This effect might be driven by an abnormal increase in the connectivity of excitatory synapses (those that drive neuronal activity) and a decrease in the connectivity of inhibitory synapses (which decrease neuronal activity). Yu and colleagues report that mice with tumours that express C420R or H1047R variants had seizures, whereas a PIK3CA variant termed $\mathrm{R} 88 \mathrm{Q}$ that does not alter synapse-related gene expression in tumours did not induce seizures. Compared with tumours expressing the $\mathrm{R} 88 \mathrm{Q}$ variant, those expressing C420R or H1047R showed an increase in the number of excitatory synapses and a decrease in the number of inhibitory synapses in tissue surrounding the mouse tumours. This pattern might explain how certain PIK3CA variants drive neuronal excitability and seizures.

When the authors engineered mouse brains to express increased amounts of the PIK3CA variants (without expressing other mutations that drive glioblastoma), they found that, in the absence of glioblastoma, H1047R drives seizures whereas $C 420 \mathrm{R}$ and $\mathrm{R} 88 \mathrm{Q}$ do not. This indicates that H1047R acts 'cell autonomously' to regulate neuronal excitability - meaning that it functions within the cell itself rather than acting on a neighbouring cell.

To determine how C420R might drive seizures, the authors expressed PIK3CA variants in a type of glial cell called an astrocyte that enhances the formation of synapses between neurons by releasing secreted molecules. The authors cultured astrocytes in vitro with neurons and assessed the astrocytes' ability to induce neuronal synapse formation. Astrocytes that expressed C420R induced higher than normal synaptic formation between neurons, whereas astrocytes that expressed H1047R had no such effect. This suggests that $C 420 R$ changes the properties of glioblastoma cells to make them induce the formation of synapses between the neurons that they contact.

Further analysis of RNA sequencing data by the authors revealed that tumour cells that expressed C420R showed increased expression of known synapse-regulating

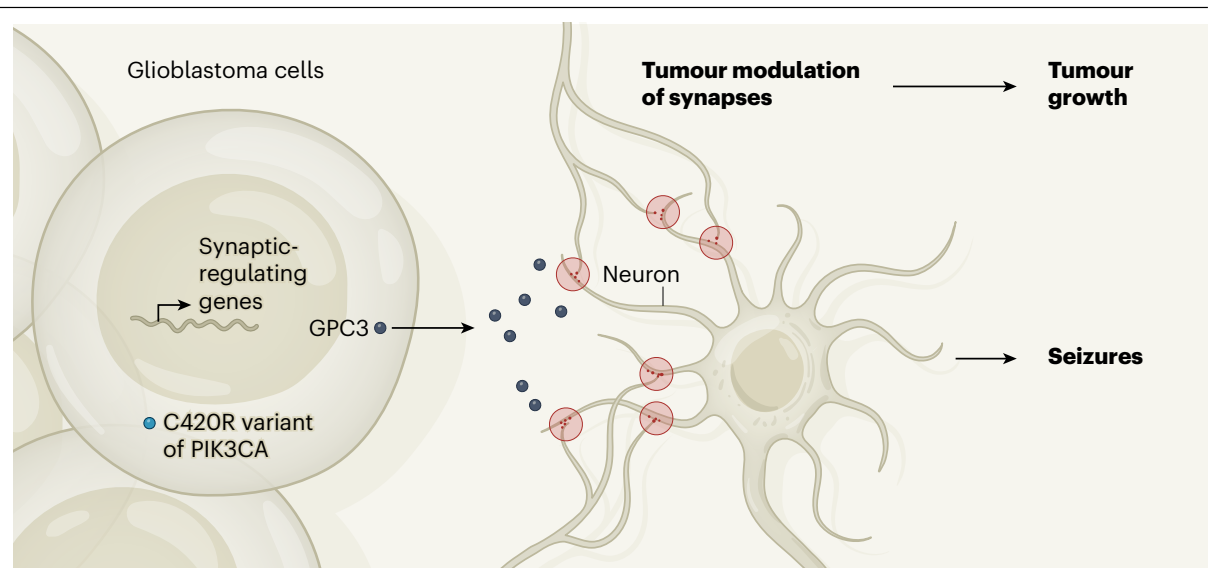

Figure 1 | Tumour modulation of neuronal connections. Yu et al. ${ }^{3}$ report their analysis of the brain cancer glioblastoma, which arises from the growth of non-neuronal (glial) cells. Using mouse models, the authors analysed mutations found in people who have glioblastoma. They report that a mutant form of the protein PIK3CA - the C420R variant - is associated with expression of genes in the cancer cell that can regulate the synaptic connections between neurons. One such gene encodes the protein glypican 3 (GPC3), which is secreted by cells and can boost synapse formation (synapses shown in pink). This leads to a rise in synaptic activity, which was associated with tumour progression. Enhanced neuronal activity might explain why seizures are associated with glioblastoma.

factors that are secreted by astrocytes. These included members of the glypican family (part of a group of sugar-containing proteins called proteoglycans), which can induce the formation of excitatory synapses in the brain?

The expression of one member of this family, glypican 3, was particularly highly upregulated in tumour cells that expressed C420R. When the authors engineered glioblastomas expressing C420R to lack glypican 3 , this caused a decrease in seizures and a longer lifespan compared with mice that had C420R-expressing glioblastomas that expressed glypican 3 . By contrast, animals with glioblastomas engineered to have higher expression of glypican 3 had more seizures and a decreased lifespan compared with animals with glioblastomas that did not overexpress glypican 3 . This suggests that glypican 3 is necessary and sufficient in the context of glioblastoma to regulate seizures by controlling synapse formation in the neuronal tissue around the tumour.

Yu and colleagues' work supports growing evidence that tumour cells interact with their neighbouring healthy cells to alter brain function. Recent work has revealed that cells of the glioblastoma itself can form a synapse with surrounding neurons (in such synapses, the tumour has postsynaptic structures and the neuron forms presynaptic structures), and that blocking this synaptic input to the glioblastoma decreases tumour growth ${ }^{8-10}$. Yu et al. now reveal how a glioblastoma can remodel connections between its neighbouring neurons, and report that the underlying mechanism differs depending on the specific PIK3CA variant involved. It will be interesting to determine in future studies whether the same synapse-promoting signals are responsible for regulating the synapses that form between a tumour and its neighbouring neurons, and for regulating synapses that form between neurons surrounding the tumour. This is an intriguing matter, because glypicans regulate neuronal synapse formation through what are known as AMPA receptors, and the synapses that form between neurons and glioblastoma cells use AMPA receptors for signalling 8,9 .

Abnormally high expression of glypican family members occurs in various types of tumour, including liver and pancreatic cancer $^{11,12}$. In those cases, glypicans are thought to bind to and regulate the signalling of growth factors that promote tumour growth. Glypicans are clearly of interest in trying to understand many disorders, and efforts being made to block their function in other cancers might suggest approaches worth testing for use in glioblastoma treatments ${ }^{11,12}$.

Nicola J. Allen is at the Salk Institute for Biological Studies, La Jolla, California 92037, USA.

e-mail: nallen@salk.edu

\footnotetext{
Noorani, I. Cancers 11, 1335 (2019).

Allen, N. J. \& Lyons, D. A. Science 362, 181-185 (2018). $\mathrm{Yu}, \mathrm{K}$. et al. Nature 578, 166-171 (2020).

Brennan, C. W. et al. Cell 155, 462-477 (2013).

5. Cancer Genome Atlas Research Network Nature 455, 1061-1068 (2008)

6. Jung, E. et al. Nature Neurosci. 22, 1951-1960 (2019). 7. Allen, N. J. et al. Nature 486, 410-414 (2012).

8. Venkataramani, V. et al. Nature 573, 532-538 (2019).

9. Venkatesh, H. S. et al. Nature 573, 539-545 (2019).

10. Barria, A. Nature 573, 499-501 (2019).

11. Li, N., Gao, W., Zhang, Y.-F. \& Ho, M. Trends Cancer 4, 741-754 (2018).

12. Nishida, T. \& Kataoka, H. Cancers 11, 1339 (2019).
} 